\title{
Development and method validation for determination of Deltamethrin residue in olive oil using a reversed-phase high performance liquid chromatography
}

\author{
Ikram Jaabiri ${ }^{1}$, Dalel Belhaj ${ }^{2 *}$, Nesrine Turki ${ }^{3}$, Monem Kallel $^{4}$, Habib Ayadi ${ }^{5}$, \\ Mohieddine Ksantini $^{6}$, Jalel Bouzid ${ }^{7}$, Radhia Gargouri ${ }^{8}$ \\ ${ }^{1-2-3-4-7}$ University of Sfax-Tunisia, ENIS. Laboratory of Water-Energy-Environment. Street Soukra Km 3.5. BP \\ 1173 CP 3038. \\ ${ }^{5}$ University of Sfax-Tunisia, Faculty of Sciences, Departement of Life Sciences, Laboratory of Biodiversity and \\ Aquatic Ecosystems, Ecology and Planktonology, Street Soukra Km 3.5 \\ BP 1171 CP 3000. \\ ${ }^{6}$ University of Sfax-Tunisia, Laboratory of Grown Plant Protection and Environment, Institute of Olivier, BP \\ 1087 CP 3000. \\ ${ }^{8}$ University of Sfax-Tunisia, ENIS. Laboratory of Plant Biotechnologies applied to crop improvement Street \\ Soukra Km 3.5. BP 1173 CP 3038.
}

\begin{abstract}
Olive oil is the most important commodities produced in the Mediterranean region. Due to its significant economical importance, the usage of pesticides in its production is systematic, by using a wide range of plant protection products with a variety of modes of action. As a consequence, monitoring of their residue levels in these products is a necessity. In the present study a reversed-phase high performance liquid chromatography method, with a short sample preparation step, based on acetonitrile extraction is developed and validated in olive oil, with a large scope that includes Deltamethrin as pesticide. Good sensitivity and selectivity of the method were obtained with limits of quantification at $0.2 \mathrm{mg} \mathrm{kg}^{-1}$. Deltamethrin has recovery rate which is of about $80 \%$. We confirm also the efficiency of alumina, used as adsorbent in the clean up step, to remove triglycerides and to get a pure extract.

The agronomic implementation of this protocol allows us to determine the influence of some parameters on the dose and the period of treatment affecting the detected quantities of Deltamethrin residues in the produced olive oil. Indeed, we prove that the treatment dose should be specific for each case considering the olive variety, the geography of the orchard, and the predicted harvest time to determine the convenient dose of treatment. In addition, the results show that the preventive treatment at the blooming phase, does not lead to the concentration of Deltamethrin residues in the oil as it happens at the lipogenesis phase.
\end{abstract}

Keywords: Clean up, Deltamethrin residues, HPLC, olive oil, dose of treatment

\section{Introduction}

Olive oil constitutes an essential ingredient in the Mediterranean meals, and more still, nowadays, we are discovering the multiple therapeutic virtues of this aliment thanks to its richness of antioxidants. Thus, producing countries are stimulated to enhance their export rate since it highly contributes in the national economy. Consequently, the use of agrochemicals and pesticides becomes compulsory to avoid the encountered production losses and in order to attain a higher quantity with a better quality.

This issue is constantly subject to environmental debates. Indeed, these agricultural practices are responsible of potential risk for human health and can be a source of contamination for the soil, the micro-flora and the groundwater, leading to the entire ecosystem imbalance.

With the emergence of the concept of sustainable development, organic food, and the expected decline of Maximum Residue Levels (MRLs) as those of baby-food, the producers are obliged to provide the external market with an olive oil containing pesticide quantities below the MRLs.

These rigorous controls require pesticide residues extraction, purification and then analysis with accuracy. Many studies pointed out the difficulties in the separation of the pesticide molecules from fatty matrix that contains between 98 and $99 \%$ of triglycerides [1], and worse still; the pesticide's components are concentrated in olive oil during the processes of its formation and extraction [2]. Besides, the oil matrix protects them from any oxidation or degradation phenomena that lead to their persistence even at low levels.

Therefore, a crucial clean up method is necessary for a precise separation of the pesticides from oil in order to avoid the co-elution of fats during chromatographic analysis. In fact, high molecular weight lipid (6001500) can cause damages on columns that may reduce the resolution's efficiency [3]. 
The low temperature technique $\left(-20^{\circ} \mathrm{C}\right)$ was used few years ago to separate fatty molecules from the solvent's fraction containing pesticide residues [4]. Recently, many protocols based on the Solid Phase Matrix Dispersion (SPMD) [5], new technology, are used for the analysis of pesticide residues in olive oil. However, their main disadvantage is the lack of automatism and time consuming [6,1]. In general, the extraction of pesticides from olive oil is based on a liquid-liquid extraction (LLE) using acetonitrile saturated with $n$-hexane $[7,3,1]$. However, many other extraction techniques are described, such as: Solid Phase Extraction (SPE), used alone or in combination with LLE [8], Supercritical Fluid Extraction (SFE); Pressurized Fluid Extraction (PFE), and soxhlet extraction. Clean up procedures are mainly based on Gel Permeation Chromatography (GPC) $[9,10]$. This universal technique can be implemented for a large range of pesticides. In addition, several adsorbents were used like silica gel, alumina and florisil for removing fats.

Analysis by Gas Chromatography (GC) has been carried out, using several types of detectors as Flame Ionization Detector FID [11], Nitrogen - Phosphore Detector NPD [12], or Electrochemical Detector ECD $[12,13,14]$. This technique has a good potential separation capacity when it is connected to MS/MS detector that enhances its sensitivity [9]. Nevertheless, it remains very expensive. Nowadays, the emergence of polar pesticides (less dangerous for the environment) allows the development of the Liquid Chromatography with Mass Spectrometry LC-MS $[7,6]$.

The pesticides belonging to the pyrethroid group, especially Deltamethrin, are the most frequently used chemicals to control several olive tree pests. Many researches focused on the study of Deltamethrin effect on microbial communities [15], on the development and the survival of species of insects [16], and on its non target impact on soil arthropods [17]. Moreover, some studies are concerned with the comparison of the effect of different formulation types of Deltamethrin on its behavior and persistence [18]. Its decrease during the commercial processing is also developed [19]. In these cases, researchers use molecular characterization [20], biological [21] and spectrophotometric quantifications with iodine titration [22].

Our study aims to develop an efficient, cheap and rapid technique for Deltamethrin residue analysis in olive oil by a reversed phase HPLC with diode array detection. Firstly, two parts are developed here namely extraction - clean up and HPLC analysis. Secondly, the set up technique will be exploited to determine the influence of some agronomic parameters (variety, locality, date of harvest) on the recovered quantities of Deltamethrin residues in the produced olive oil. Moreover we will study the influence of the preventive treatments (at the blooming) compared to the curative ones (at the lipogenesis).

\subsection{Raw materials and reagents}

\section{Experimental}

The analytical standard of Deltamethrin PESTANAL ( $99.8 \%$ of purity). The HPLC grade solvents used in these experiments (dichloromethane, hexane and methanol) are obtained from Sigma-Aldrich's Fluka (Riedel DE Haen, Germany). Acetonitrile is procured from Pharmburg (Hambourg, Germany). Pure Anhydrous sodium sulfate of analytical grade is purchased from Labosi, Osi laboratories (Paris, France). Alumina, Florisil, and Silica gel are supplied from E. Merck (Dramstadt, Germany). The water used for HPLC's mobile phase is of high purity (18 M..cm).

Olive trees' treatment is done with solutions of DECIS (Emulsible Concentrate of the commercial insecticide containing $10 \%$ of Deltamethrin) bought from Bayer CropScience.

Individual stocks of standard solutions of Deltamethrin $\left(200 \mathrm{mg} \mathrm{L}^{-1}\right.$ and $\left.20 \mathrm{mg} \mathrm{L}^{-1}\right)$ are prepared by weighting and dissolving the powder in methanol. They are stored in glass bottles at $-18^{\circ} \mathrm{C}$, in the dark.

Working standard solutions are prepared by dilution in methanol, and then stored at $\left(4^{\circ} \mathrm{C}\right)$.

During the whole experiment, no degradation of the pesticide is detected under the storage conditions.

Micro-columns, used to purify Deltamethrin residues, are prepared in the laboratory using $350 \mathrm{mg}$ of activated alumina 90 per gram of olive oil [14].

Olive oil samples are obtained from different varieties of olive fruits (Chemleli Sfax, Zalmati Zarzis, Chetoui, Chemcheli Gafsa) collected from different places (Chaffar, Boughrara, Zaghden) in Sfax region (south east of Tunisia) and treated with different quantities $\left(1 \mathrm{ml} \mathrm{L}^{-1}\right.$ and $\left.1.5 \mathrm{ml} \mathrm{L}-1\right)$. All the samples are stored at $4^{\circ} \mathrm{C}$ in glass bottles.

\subsection{Sample preparation}

The sample processing [23] is described as follow: briefly, pretreatment of olive oil samples is performed by adding $115 \mu \mathrm{L}$ of standard solution of Deltamethrin at $20 \mathrm{mg} \mathrm{L}^{-1}$ to $2 \mathrm{~g}$ of olive oil (corresponding to $2.3 \mathrm{~mL}$ ). The final concentration is of about $1 \mathrm{mg} \mathrm{L}^{-1}$. These components are homogenized for 1hour under a slow agitation.

Samples of olive oil, supplemented with Deltamethrin, are dissolved in $10 \mathrm{~mL}$ of acetonitrile and $2 \mathrm{~mL}$ of hexane. Then, $4 \mathrm{~g}$ of anhydrous sodium sulfate are added. The mixture is stirred at $400 \mathrm{rpm}$ for $45 \mathrm{~min}$, and then the solution is transferred to a separation funnel of $60 \mathrm{~mL}$. 
After one hour of decantation, the light phase corresponding to the acetonitrile fraction is separated and transferred into a $10 \mathrm{~mL}$ flask. The acetonitrile is then evaporated to nearly dryness in a water bath at $30{ }^{\circ} \mathrm{C}$ and under a low nitrogen stream. The residue was dissolved in $2 \mathrm{~mL}$ of acetonitrile.

Micro-columns are prepared using activated alumina 90; by placing in a Pasteur pipette a small piece of glass wool and $700 \mathrm{mg}$ of the adsorbent. This support is wetted with $3 \mathrm{~mL}$ of acetonitrile. The concentrated extract is then injected in the micro-column. The elution is done with 3 fractions of $1 \mathrm{~mL}$ of acetonitrile. The first fraction resulting from the injection of the extract is not collected. The recovery of aliquots began after the injection of the first milliliter of elution's solvent. Then, the pure extract is directly taken for HPLC analysis to avoid any expected degradation of Deltamethrin.

\subsection{HPLC analysis}

Hewlett Packard model 1100 HPLC equipped with a Spherisorb ODS column $5 \mu$ m (diameter 6 x 250 $\mathrm{mm}$ ) and UV diode array detector (DAD) installed with an Agilent Chemstation 1990 - 2000 software, is used for data acquisition, integration and processing.

As the analysis of Deltamethrin by HPLC in olive oil was not - into our knowledge- described in the literature, we intend to determine the nature and the composition of an adequate mobile phase, its flow rate, and the sample's injection volume. To reach these purposes, a standard solution of commercial Deltamethrin, at a given concentration is used. Moreover, several organic solvents are tested at different flow rates. The behavior of chromatograms of Deltamethrin changes by changing the nature and the polarity of the mobile phase.

When optimal conditions are set, a calibration curve is established to define the linearity of the developed method, in the range of concentrations between 0.2 and $30 \mathrm{mg} \mathrm{L}^{-1}$.

Then, all analyses carried out in our experiments are done at the selected optimal conditions under the maximum of UV absorption of Deltamethrin that is defined by the DAD and confirmed by the UV- spectrophotometer.

\section{Results and discussion}

In order to evaluate the performance of the method, an optimization of the experimental conditions for HPLC analysis, is performed using standard solution of Deltamethrin. The selected conditions are used to evaluate the efficiency of the extraction-clean up procedure. The whole selected experimental conditions are applied to the analysis of Deltamethrin in different samples of olive oil obtained from treated trees.

\subsection{Optimization of HPLC analysis conditions}

\subsubsection{Qualitative analysis}

To establish the best conditions of the HPLC analysis of Deltamethrin, samples of pure olive oil supplemented with about $1 \mathrm{mg} \mathrm{L}^{-1}$ of Deltamethrin are used. Before injection, samples are purified by passing through an alumina column.

The HPLC analyses are performed under the maximum absorption's wavelength (206 nm) of Deltamethrin determined by the diode array detector and a UV- spectrophotometer.

Two mobile phases (hexane - ethanol and methanol - water) are tested in gradient mode to determine an optimal composition giving a satisfactory compromise between resolution and analysis time.

The chromatograms given by the first mobile phase are not stable over the time. This instability is explained by the high volatility of hexane. In the contrary, the chromatograms obtained with the methanol - water mixture are stable and the corresponding peaks are well separated without any interference with the fatty molecules (Fig. 1). After testing different parameter levels, the following chromatographic conditions: a mobile phase: methanol water: $90 \%-10 \%$, a flow rate of $0.8 \mathrm{ml} / \mathrm{min}$ and an injection volume of $10 \mu \mathrm{l}$ are selected.

Under these conditions the chromatograms shown in fig1 is obtained. It clearly demonstrates the efficiency of the selected optimal conditions since the peaks are well separated and the analysis time is relatively short. 


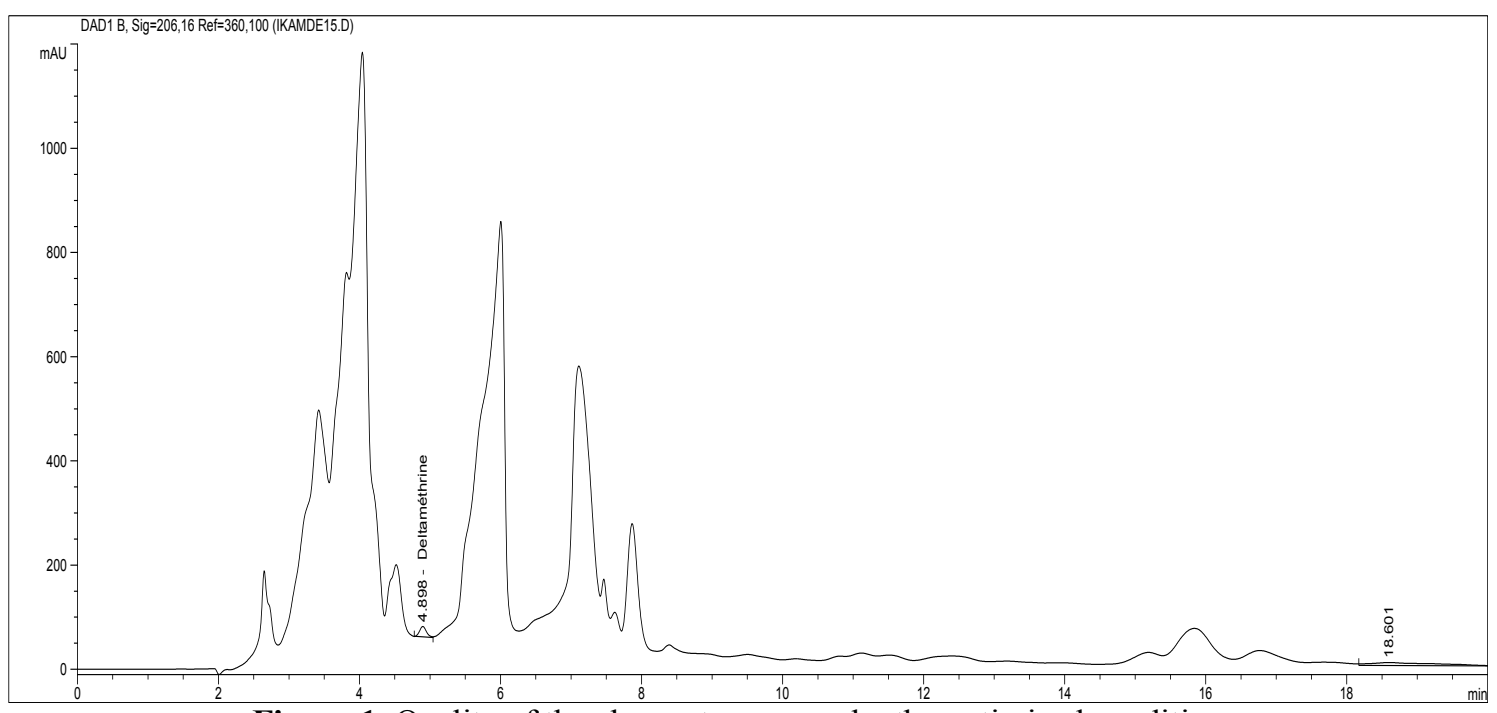

Figure 1. Quality of the chromatogram under the optimized conditions

\subsubsection{Quantitative analysis}

3.1.2.1. Calibration curve

A calibration curve is established using standard solutions of Deltamethrin (Fig. 2) of a concentration ranging between 0.2 and $30 \mathrm{mg} \mathrm{L}^{-1}$.

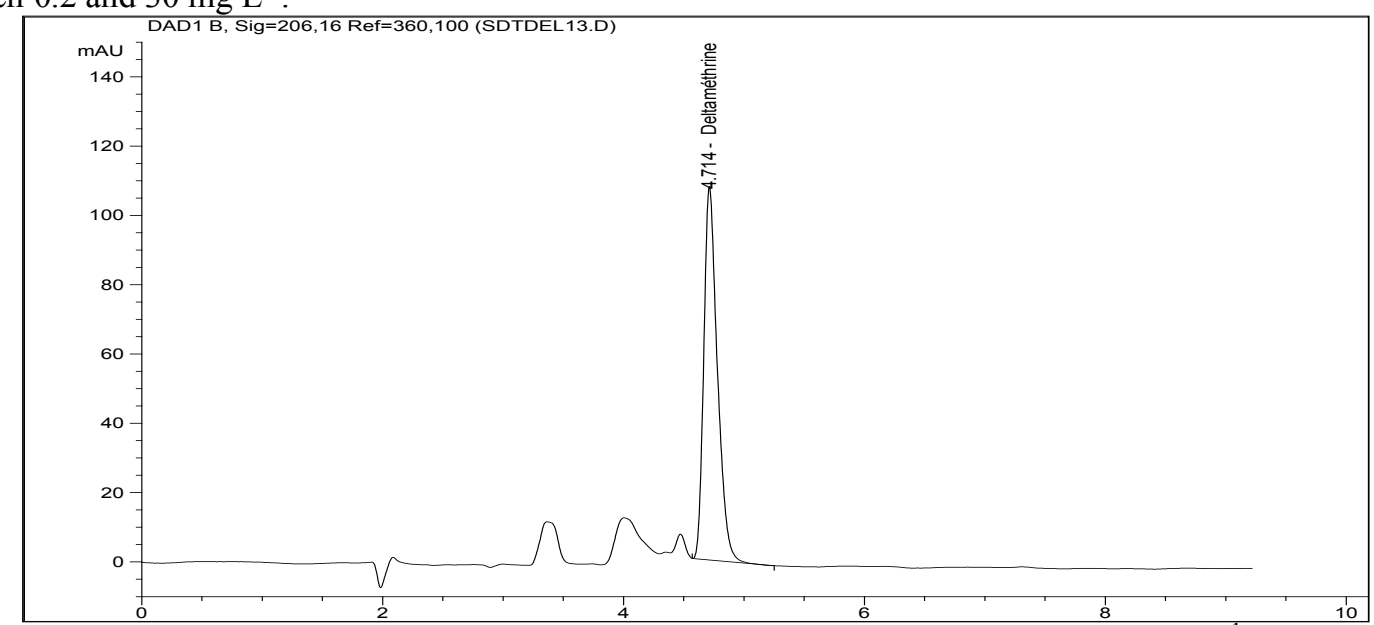

Figure 2. Chromatogram of the standard solutions of Deltamethrin $\left(10 \mathrm{mg} \mathrm{kg}^{-1}\right)$

the linearity is clearly observed (Fig. 3). The correlation coefficient obtained under these conditions is of $0.9996>0.995$ [24].

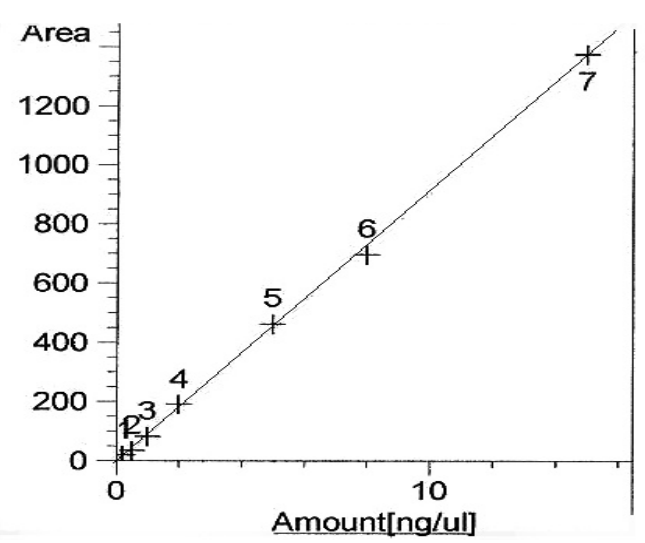

Deltaméthrine at exp. RT: 4.805

DAD1 B, Sig=206,16 Ref $=360,100$

Correlation:

0.99962

Residual Std. Dev.: 14.21041

Formula: $y=m x+b$

$\mathrm{m}: \quad 91.12226$

b: $\quad-5.16228$

$x$ : Amount[ng/ul]

$y$ : Area

Figure 3. Calibration curve 


\subsubsection{Detection and quantification limits}

These two parameters are determined according to AFNOR standards [24]. The Detection Limit (DL) (signal to noise ratio 1:3) is observed to be $0.08 \mathrm{mg} \mathrm{kg}^{-1}$.

The Quantification Limit (QL) is determined by the following formula: $\mathrm{QL}=3 \times \mathrm{DL}$. It is nearly $0.2 \mathrm{mg} \mathrm{kg}^{-1}$. These values below the MRL of Deltamethrin in olive oil $\left(1 \mathrm{mg} \mathrm{kg}^{-1}\right)$ further confirm the efficiency of the quantification technique developed for this type of matrix.

\subsubsection{Repeatability}

The repeatability of the method is determined by measuring the variation coefficient (the ratio of the mean value to its standard deviation) for the pesticide at a given concentration ( $1 \mathrm{mg} \mathrm{kg}^{-1}$ of Deltamethrin) extracted from olive oil during three successive days. The obtained value is of nearly $10 \%$.

\subsection{Optimization of the extraction -clean up step}

This step is considered as crucial since it determines the quality of chromatograms and the performance of all the elaborated process. Tests are carried out on oil samples supplemented with a standard solution of Deltamethrin

A variety of solvents (dichloromethane, n-hexane, acetonitrile ...) and different adsorbents (Florisil, gel silica, alumina) are tested for the extraction-clean up steps.

In order to evaluate the performance of the extraction-clean up procedure, the recovery rate is determined by HPLC. Chromatographic results show a value of about $80 \%$, when acetonitrile - hexane solvent is used. This level corroborate with the French ministry of health [25] indicating that this rate should ranges between $60 \%$ and $120 \%$. Thus, the obtained value confirms the efficiency of the mixture acetonitrile - hexane to extract Deltamethrin (Fig. 4).

It, also, show the efficiency of alumina to remove fat molecules. The elimination of fatty substances is improved by increasing the weight of alumina from 250 to $350 \mathrm{mg}$ per gram of olive oil. This result agree with that of Telling et al [26] who indicate that an excess of alumina is required in the clean up procedure.

Our results confirm those found by Lentza Rizos et al [14]. However, they are contradictory to those described in the bulletin of CAMRPF [27] indicating that the clean up on alumina is effective for $\alpha$-endosulfan but not for Deltamethrin extraction from foodstuffs.

Each sample is purified on its own microcolumn to prevent the inactivation stage of alumina and to avoid the clogging risks.

Owing to the low volume of the extracts, their concentration is carried out by nitrogen bubbling through the extract placed in a water bath at $30^{\circ} \mathrm{C}$, rather than the concentration by rotavapor.

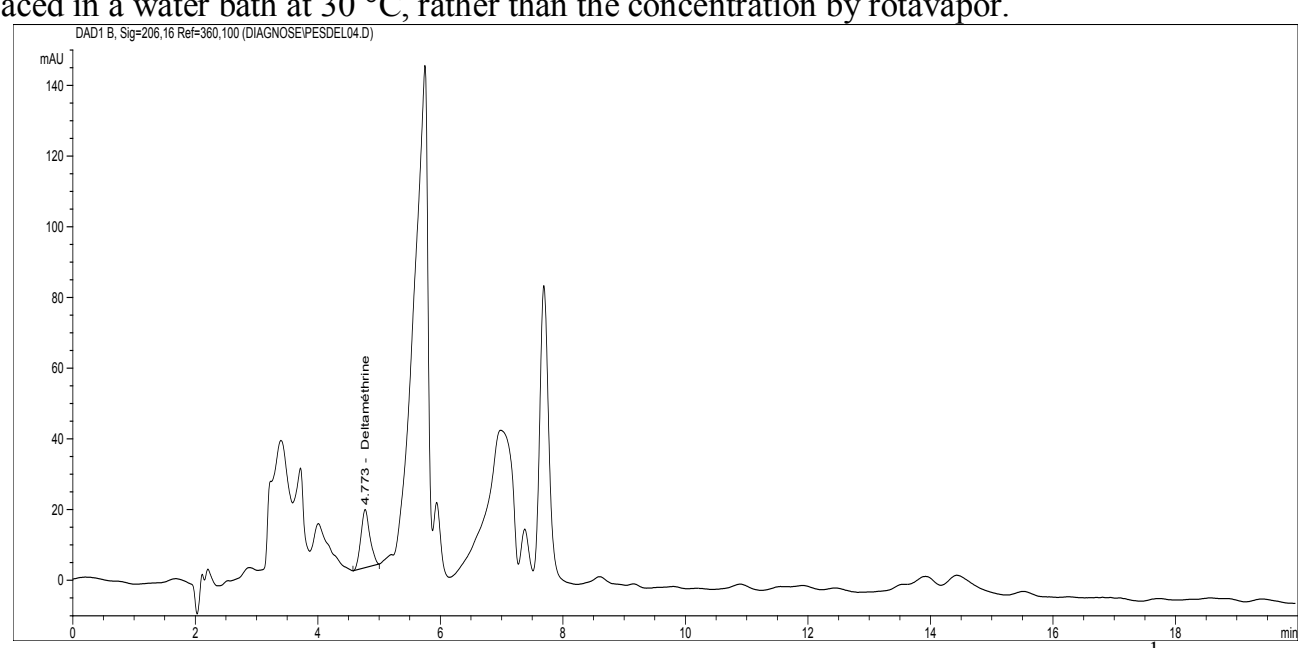

Figure 4. Chromatogram of extracted residue from fortified oil $\left(1 \mathrm{mg} \mathrm{kg}^{-1}\right)$

\subsection{Agro-environmental application}

The optimized protocol results in well separated and well resolved chromatograms with a minimum of interference between the different compounds, in a short time of analysis. Therefore, it would be interesting to use this technique for analysis of parameters influencing the residual quantities of pesticides namely the variety, the locality, the vegetative stage of the trees (blooming and lipogenesis), and the period between the treatment and the harvest. 


\subsubsection{Effect of preventive treatment at the flowering phase}

Samples of olive oil are obtained from the orchard Zaghden which is treated at the flowering phase. This preventive treatment used to avoid the psyllid is organized yearly by the state. The sampling is performed 6 months later, at the end of the lipogenesis phase (at the harvest moment).

The results show that oil samples do not contain any traces of Deltamethrin residues. These data can be explained by the fact that the treatment at the blossoming phase does not lead to the storage of this fat-soluble molecule in the resulting fruits because of the absence of a direct contact with the olive fruit itself. We can also suggest that the period of 6 months may be sufficient to degrade the Deltamethrin molecule, so the residual quantity in the fruit drop below the detection limit.

However, samples of the orchard Boughrara or Chaffar, that are treated curatively at the fructification stage (or lipogenesis), tend to accumulate Deltamethrin residues.

\subsubsection{Effect of the curative treatment on pendant fruits}

In order to monitor the Deltamethrin molecule over the time, olive trees of the variety Chemleli Sfax (from the orchard Chaffar) are treated by terrestrial spraying of a solution of DECIS (a Deltamethrin - based insecticide), at a concentration of $1 \mathrm{ml} \mathrm{L}^{-1}$. Sampling is conducted every three days after treatment.

The results (Fig. 5) show that residual amounts of Deltamethrin decline slowly over time.

These data show that after two weeks of treatment, the rate of Deltamethrin residue detected in the oil, remains high and above the MRL (about $2 \mathrm{mg} \mathrm{kg}^{-1}$ ). One further week is needed to reach a rate of Deltamethrin below the MRL (about $0.8 \mathrm{mg} \mathrm{kg}^{-1}$ ).

Therefore, these results call into question the time of harvest (major parameter for the approval of pesticides) predicted in the datasheet of the commercial product ( 7 days). These results suggest that preventive measures must be taken into account in order to protect consumer's health

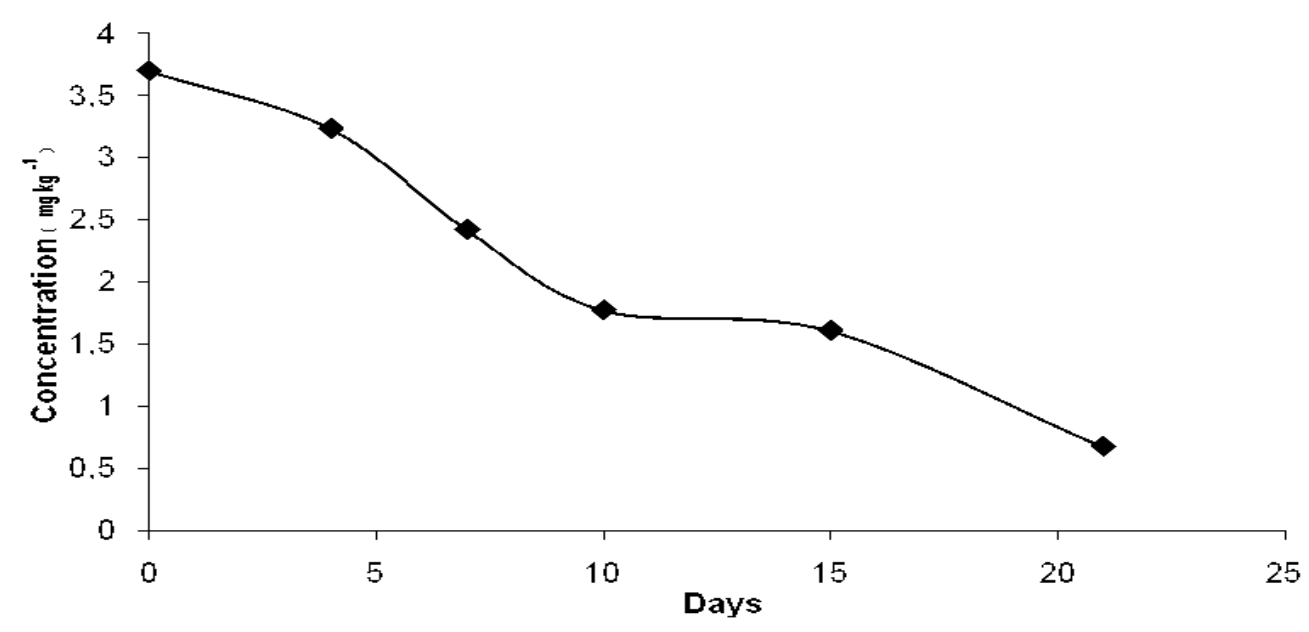

Figure 5. Kinetic of degradation of Deltamethrin

\subsubsection{Effect of the dose and the geography on the residual quantities of Deltamethrin}

Analyses of Deltamethrin residues are carried out on oil samples of the same variety Chemlali Sfax. Samples differ in the locality and in the dose of treatment. In fact, samples obtained from the orchards Boughrara and those from Chaffar are treated with doses of 1.5 and $1.0 \mathrm{ml} \mathrm{L}^{-1}$ respectivly. Sampling is done in the same conditions twenty days later.

The results (Table 1) show a significant difference in the quantities of detected residue in the oil samples. Added to the induced overdose of $0.5 \mathrm{~mL} \mathrm{~L}^{-1}$, the accidental ones (bottom of bowl, excessive dose, carelessness of the workers...) can contribute to increase the amount of residual Deltamethrin.

Table 1. Comparison of the detected quantities in samples of the variety Chemleli Sfax obtained from two different orchards

\begin{tabular}{cccc}
\hline Locality & $\begin{array}{c}\text { Treatment's dose } \\
\left(\mathbf{~ m L ~ L ~}^{-1}\right)\end{array}$ & $\begin{array}{c}\text { Period separating treatment } \\
\text { and sampling (days) }\end{array}$ & $\begin{array}{c}\text { Average of detected quantities } \\
\left(\mathbf{m g ~ k g}^{-1}\right)\end{array}$ \\
\hline Chaffar & 1.0 & 21 & 0.54 \\
Boughrara & 1.5 & 21 & 3.70 \\
\hline
\end{tabular}


It seems that the fact that the orchard Chaffar is close to the sea may contribute to decrease Deltamethrin residual amounts in olives. This may be due to the sea winds and the night humidity ( $>0.2 \mathrm{~mm}$ per day) that are likely to leach the insecticide deposed on the tree.

The comparison between the detected quantities shows that 21 days are enough to drop the residue of Deltamethrin below the MRL for the oil of the orchard Chaffar. However, for the insider zone (Boughrara), this amount remains higher than the maximum limit. We can conclude that the deadline for harvest after treatment should not be the same for olive oil provided from different localities.

\subsubsection{Sensitivity of some varieties of olive tree to the treatment with Deltamethrin}

This study focused on 4 varieties from the orchard of Boughrara. The obtained results of samples' analysis (table 2) show the detection of Deltamethrin in both varieties Chemleli Sfax and Zalmati Zarzis, but with a huge difference in the quantities. Indeed, in the olive oil of Chemleli Sfax we detect more than the triple of the MRL $\left(1 \mathrm{mg} \mathrm{kg}^{-1}\right)$.

As a conclusion, the retention of Deltamethrin in the pulp fluctuates depending on the olive tree variety. Indeed, the Chemlali Sfax variety seems to be more sensitive as it shows the highest levels of Deltamethrin residues.

This result may be due either to a higher proportion of oil in the pulp, or to the texture of the fruit itself and its capacity to fix Deltamethrin molecule.

Moreover, according to Lopez Villata [28], this result can be explained by the negative correlation between the weight of the fruit, which is a varietals characteristic, and the detected quantities of Deltamethrin (mg of Deltamethrin per kg of fruit).

Table 2. Quantities of Deltamethrin residue detected in different varieties

\begin{tabular}{|c|c|c|}
\hline Variety & $\begin{array}{c}\text { Fruit's average weight } \\
\text { (g) }\end{array}$ & Average of detected residue $\left(\mathrm{mg} \mathrm{kg}^{-1}\right)$ \\
\hline Chemleli Sfax & 1.00 & 3.70 \\
\hline Zalmati Zarzis & 1.50 & 0.28 \\
\hline Chetoui & 2.00 & $<\mathrm{DL}$ \\
\hline Chemchali Gafsa & 2.26 & $<\overline{D L}$ \\
\hline
\end{tabular}

\section{Conclusion}

This report enables to develop an analytical tool for detection of Deltamethrin residues in olive oil. Indeed, the developed protocol is both effective and economical, allowing the use of very small amounts of solvents and chemicals.

This technique is also reproducible. In addition, the method of purification is quite selective, generating a good separation of the peaks and avoiding the risk of interference.

This procedure can be introduced into the conventional analytical laboratories concerned with the quality of olive oil to assess its compliance with the standards.

Moreover, in this study, we proved the slow degradation of Deltamethrin and its bioaccumulation in fatty matrices like olive oil. This explains that to use Deltamethrin for fighting pests, it is recommended to lengthen treatments deadlines, or to avoid its use during lipogenesis.

In addition, it should be taken into consideration the effect of the proximity to the sea and the weight of olive fruits, which is a variety characteristic on the residual quantities of Deltamethrin. The treatment's dose should be studied according to these factors; hence we can reach a stable mg per $\mathrm{kg}$ ratio leading to a pesticide's residue below the MRL.

Finally, we confirmed the efficiency of the preventive treatment of olive trees by Deltamethrin without engendering any traces of residues. AFNOR (1999). Eau - contrôle qualité, T90 Q.[2] $\quad$ E. G. Amvrazi and T. A. Albanis, Determination of various pesticide
residues in Greek virgin olive oil by a multi-residue method using different clean up procedures and gas chromatography. T.D. Lekkas (Editor). 9 th International Conference in Environmental Science Technology. University of the Aegean, Rhodes Island, Greece, 2005

[3] M. A. Aramendia, F. Lafont, A. Marinas, J. M. Marinas, J. M. Moreno, J. M. Porras and F. Jurbano, Determinacion de herbicidas en aceite de oliva mediante GC-MS y/o HPLC-MS. Symposium Cientıfico-Ténico EXPOLIVA. Spain, 2005.

[4] C. A. Badji, R. N. Guedes, A. A. Silva, A. S. Corre, M. E. Queiroz and M. Michereff-Filho, Non-target impact of deltamethrin on soil arthropods of maize fields under conventional and no-tillage cultivation. Journal of Application Entomology, 2007, 131[1], 5058 .

[5] E. Ballesteros, A. Garcia sanchez and N. Ramos Martos, Simultaneous multidetermination of residues of pesticides and polycyclic aromatic hydrocarbons in olive and olive-pomace oils by gas chromatography/tandem mass spectrometry, Journal of Chromatography A, 2006, 1111, 89-96.

[6] E. Ballesteros Tribaldo, Carbamate pesticide residues in food. Hand book of Food Analysis. Marcel Dekker, New York, 2004, 1177-1209. 
[7] R. Boussahel, K. M. Moussaoui and D. Harik, Determination of residues of deltaméthrin in wheat and potato by HPLC, African Journal of Agriculture Research, 2006, 1 [5], 182-185.

[8] CAMRPF. Committee of the analytical methods for residues of pesticides in foodstuffs of the Ministry of Agriculture. Fisheries and Foods. Analyst. 1992, 117, 1451 .

[9] M. J. Chavarri, A. Herrera and A. Arin, The decrease in pesticides in fruit and vegetables during commercial processing, International Journal of Food Science Technology, 2005, 40, 205-211.

[10] A. Di Muccio, P. Pelosi, D. A. Barbini, T. Generali, S. Girolimetti, P. Stefanelli, A. Leonelli, G. Ama'ndola, L. Vergori and E. V. Fresquet, Determination of pyrethroid pesticide residues in fatty materials by solid-matrix dispersion partition, followed by minicolumn size-exclusion chromatography, Journal of Chromatography A, 1999, 833,19-34.

[11] A. A. Enayati, J. G. Vontas, G. J. Small, L. M. Carroll and J. Hemingway, Quantification of pyrethroid insecticides from treated bednets using a mosquito recombinant glutathione S-transferase, Med. Vet. Entom. 2001, 15, 58-63.

[12] J. L. Fernandez Moreno, F. J. Arrebola Liébanas, A. Garrido Frenich and J. L. Martinez Vidal, Evaluation of different sample treatments for determining pesticide residues in fat vegetable matrices like avocado, by low-pressure gas chromatography-tandem mass spectrometry, Journal of Chromatography A, 2006,1111, $97-105$.

[13] C. Ferrer, M. J. Gomez, J. F. Garcia-Reyes, I. Ferrer, E. M. Thurman and A. R. Fernandez-Alba, Determination of pesticide residues in olives and olive oil by matrix solid-phase dispersion followed by gas chromatography/mass spectrometry and liquid chromatography/tandem mass spectrometry, Journal of Chromatography A, 2005, 1069, 183 - 194.

[14] J. F. Garcia-Reyes, C. Ferrer, M. J. Gomez-Ramos, A. Molina-Diaz and A. R. Fernandez-Alba, Determination of pesticide residues in olive oil and olives, TrAC, Trends Analytical Chemical, 2007, 26, 239-251.

[15] A. Garcia Sanchez, N. Ramos Martos and E. Ballesteros, Multiresidue analysis of pesticides in olive oil by gel permeation chromatography followed by gas chromatography -tandem mass-spectrometric determination, Analytical Chimical Acta, 2006, 558, 53-61.

[16] K. Graham, N. Mohammed, H. Rehman, A. Nazari, M. Ahmad, M. Kamal, O. Skovamand, P. Guillet, R. Allan, N. Zaim, A. Yates, J. Lines and M. Rowland, Insecticide-treated plastic tarpaulins for control of malaria vecors in refugee camps. Med. Vet. Entom. 2002, 16, 404-408.

[17] M. Guardia-Rubio, M. L. Fernandez-De Cordova, M. J. Ayora-Canada and A. Ruiz-Medina, Simplified pesticide multiresidue analysis in virgin olive oil by gas chromatography with thermoionic specific, electron-capture and mass spectrometric detection, Journal of Chromatography A, 2006, 1108, 231-239.

[18] M. L. Hopper, Automated one-step supercritical fluid extraction and clean-up system for the analysis of pesticide residues in fatty matrices, Journal of Chromatography A, 1999, 840, 181-188.

[19] M. Jawara, M. Pinder, B. G. Cham, Walraven and J. Rowley, Comparison of deltamethrin tablet formulation with liquid deltamethrin and permethrin for bednet treatment in The Gambia, Tropical Medicine and International Health, 2001, 6 [4], $309-316$.

[20] C. W. Knapp, T. Caquet, M. L. Hanson, L. Lagadic and D. W. Graham, Response of water column microbial communities to sudden exposure to deltamethrin in aquatic mesocosms, FEMS Microbiology Ecology. 2005, 54,157-165.

[21] C. H. Lenza-Rizos, E. J. Avramides and E. Visi, Determination of residues of endosulfan and five pyrethroid insecticides in virgin olive oil using gas chromatography with electron-capture detection, Journal of Chromatography A, 2001, 921, 397-304.

[22] M. C. Lopez-Villalta, Contrôle des parasites et des maladies de l'olivier. L.R.Cuellar, Espagne, D.L. 1999, M-9362.

[23] D. Martinez-Torres, F. Chandre, M. S. Williamson, F. Darriet, J. B. Bergé, A. L. Devonshire, P. Guillet, N. Pasteur and D. Pauron, Molecular characterization of pyrethroid knockdown resistance (Kdr) in the major malaria vector Anopheles gambiae, Insect Molecular Biology, 1998, 7 [2], 179-184.

[24] Ministry of Health, Family and the Disabled. Method of application of the order on methods of analysis of water samples and their performance characteristics. Official Bulletin No.49. France, 2003.

[25] K. Patel, R. J. Fussell, M. Hetmanski, D. M, Goodall and B. J. Keely, Evaluation of gas chromatography-tandem quadrupole mass spectrometry for the determination of organochlorine pesticides in fats and oils, Journal of Chromatography A, 2005, 1068, 289 296.

[26] G. M. Telling, D. J. Sissons and H. W. Brinkmann, Determination of organochlorine insecticide residues in fatty foodstuffs using a clean-up technique based on a single column of activated alumina, Journal of chromatography, 1977, 137, $405-412$.

[27] C. Tsoutsi, I. Konstantinou, D. Hela and T. Albanis, Screening method for organophosphorus insecticides and their metabolites in olive oil samples based on headspace solid-phase micro-extraction coupled with gas chromatography, Analatycal Chemical Acta, 2006, 53-574.

[28] K. G. Wardhaugh, B. C. Longstaff and M. J. Lacey, Effects of residues of Deltamethrin in cattle faeces on the development and survival of three species of dungbreeding insect, Aust. Vet. J,1998, 76 [4], 273 - 280. 